\title{
Salt and salted food intake and subsequent risk of gastric cancer among middle-aged Japanese men and women
}

\author{
S Tsugane*,', S Sasazuki', M Kobayashi' and S Sasaki' for the JPHC Study Group ${ }^{\dagger}$ \\ 'Epidemiology and Biostatistics Division, National Cancer Center Research Institute East, 6-5-I Kashiwanoha, Kashiwa, 277-8577, Japan
}

\begin{abstract}
Evidence on the association between salt intake and gastric cancer is sparse, especially in prospective studies. We conducted a population-based prospective study in Japan, where the majority of men has been infected with Helicobacter pylori. A total of I 8684 men and 20381 women aged 40-59 years who reported their dietary habits and did not report any serious disease at baseline were followed from 1990 to 200 I. A total of 486 cases, 358 men and 128 women, with histologically confirmed gastric cancer were documented among them. The quintile category of salt intake was dose-dependently associated with gastric cancer risk in men after adjusting for potential confounding factors $(P$ for trend $<0.00 \mathrm{I})$, while a trend was not clear in women $(P$ for trend $=0.48)$. Although stratification by study area, with varied salt intake and gastric cancer incidence, attenuated the observed clear associations with salt and salted foods, the frequency categories of highly salted foods such as salted fish roe and salted fish preserves were strongly associated with the risk in both sexes. Restriction of salt and salted food intake is a practical strategy to prevent gastric cancer in areas with high risk.
\end{abstract}

British Journal of Cancer (2004) 90, 128 - 134. doi:10.1038/sj.bjc.660 I5I I www.bjcancer.com

(C) 2004 Cancer Research UK

Keywords: diet; prospective studies; salts; stomach neoplasms

Gastric cancer is the second most frequent cause of cancer deaths worldwide, with an estimated 776000 deaths in 1996, almost two-thirds of which were in developing countries (World Health Organization, 1997). In Japan, gastric cancer killed 50000 people in 2000, which was the second most frequent cancer death (17\% of all cancer deaths; Ministry of Health, Labor and Welfare, Japan). Moreover, a total of 100000 new cases of gastric cancer were estimated in Japan in 1996, and it was the most common cancer site ( $21 \%$ of all incident cancers; Research Group for Population-based Cancer Registration in Japan, 2002). Therefore, prevention of gastric cancer is one of the most important elements in any cancer control strategy both in Japan and around the world.

Although salt and salted foods are probable risk factors, based on evidence from a large number of case-control and ecological studies (Tsugane et al, 1992b; Joossens et al, 1996; Kono and Hirohata, 1996), evidence from prospective investigation is scarce and inconsistent (Nomura et al, 1990; Kneller et al 1991; Kato et al, 1992). The recent report of a joint WHO/FAO Expert Consultation concluded that 'Salt-preserved foods and salt probably increase the risk of stomach cancer' (World Health Organization, 2003). The difficulties in estimating the intake of salt per se may account for divergent findings.

We tested this hypothesis in Japan in a population-based prospective study in four public health centre areas with varying

*Correspondence: Dr S Tsugane; E-mail: stsugane@east.ncc.go.jp

† Study Group members are listed in the appendix.

Received 26 August 2003; revised 10 October 2003; accepted 15 October 2003 gastric cancer mortality rates and where the majority (75\%) of randomly selected men aged 40-49 years has been infected with Helicobacter pylori (Tsugane et al, 1994).

\section{MATERIALS AND METHODS}

\section{Study cohort}

The Japan Public Health Center-based prospective study on cancer and cardiovascular diseases (JPHC Study) Cohort I, which was partly reported elsewhere (Tsugane et al, 1999), provided a basis for this investigation. As of 1 January 1990, we established a population-based cohort of 54498 people (27062 men and 27436 women) who resided in 14 administrative districts supervised by four regional public health centres (PHCs): 12291 from Ninohe City and Karumai Town in the Ninohe PHC area of Iwate prefecture, 15782 from Yokote City and Omonogawa Town in the Yokote PHC area of Akita, 12219 from eight districts of MinamiSaku county in the Saku PHC area of Nagano and 14206 from Gushikawa City and Onna Village in the Ishikawa PHC area of Okinawa. All were born between 1930 and 1949 (40-59 years of age at the baseline). These PHC areas were selected to represent the extent of variation in the mortality rate of stomach cancer based upon our previous ecological study (Tsugane et al, 1992a; Tsubono et al, 1997). The institutional review board of the National Cancer Center approved this study.

\section{Baseline survey}

In 1990, a self-administered questionnaire was distributed to all registered residents. They were asked to report on their socio- 
demographics, personal medical history, smoking and drinking history and diet. A total of 43149 subjects, 20665 men (76\%) and 22484 women $(82 \%)$, returned their questionnaires. Although the date of questionnaire completion ranged from January 1990 to May 1992, 54\% responded between February 1990 and March 1990. Only 4\% were completed after October 1990.

The weekly intake frequency of 27 food items was reported in four categories: rarely, 1-2 days week ${ }^{-1}, 3-4$ days week $^{-1}$ and almost daily $\left(5+\right.$ days week $\left.{ }^{-1}\right)$. For rice, miso soup and nine kinds of beverage, the daily amount consumed was also reported. Design and participation rate of the baseline survey and dietary habits among JPHC study participants at baseline survey were reported elsewhere (Tsugane and Sobue, 2001; Tsugane et al, 2001).

Based on dietary record data, we developed a food composition table that corresponded to the food items listed in the questionnaire and determined standard portions/units for each food category using the observed median values. The food composition table was developed and standard portions/units were assigned separately for men and women. Daily nutrient intakes were calculated by multiplying the consumption frequency of each food by the nutrient content of the standard portions/units and summing these values for all foods. Calculation of total energy and 30 nutrients was done with the Standard Tables of Food Composition (Science and Technology Agency, 1982) and other sources. The validity was assessed among subsamples ( 94 men and 107 women) with actual nutrient intake, which was calculated based on a 28-day dietary record (7 consecutive days in four seasons; 14 days in the Ishikawa PHC area; Tsugane et al, 2003). The Spearman rank correlations between two indices for sodium intake were 0.49 in men and 0.54 in women (Tsubono et al, 2003). Those with two-time 24-h urine excretion level ( 32 men and 57 women, except Ishikawa PHC area) were 0.38 in men and 0.12 in women (Tsubono et al, 2003).

The following food items were considered as salted food in our analysis: miso soup (salt content: 0.5-1.2\%), pickled vegetables (1-10\%; specific foods include shiozuke, with $1.7 \%$ in Chinese cabbage, $2.8 \%$ in cucumber, and nuka-misozuke, with $1.7 \%$ in eggplant, $2.8 \%$ in cucumber), salted fish roe (tarako, salted Alaska pollack roe, $6.6 \%$, suziko or ikura, salted salmon roe, 9.7\%), salted fish preserves (shiokara, salted fish guts, $11.4 \%$ in squid, neri-uni, salted gonad paste of sea urchin, $11.9 \%$ ) and dried or salted fish (mezashi, salted and semidried skewered sardine, 4\%, and shio-sake, salted salmon, 8\%). Salt content data for specific food items were taken from with Standard Tables of Food Composition (Science and Technology Agency, 1982). The Spearman rank correlations with the dietary records $\left(\mathrm{g} \mathrm{day}^{-1}\right.$ ) were 0.41 for miso soup, 0.67 for pickled vegetables, 0.61 for salted fish roe, 0.43 for salted fish preserves and 0.55 for dried or salted food in men, and 0.49 , $0.61,0.47,0.34$ and 0.33 in women, respectively (Tsugane et al, 2003). Miso soup was categorised into the following four groups in this analysis: not daily, 1 cup day ${ }^{-1}, 2$ cups day ${ }^{-1}, 3+$ cups day $^{-1}$.

We excluded subjects with a self-reported serious illness (cancer, cerebrovascular disease, myocardial infarction, or chronic liver disease) at baseline as well as non-Japanese subjects and subjects who had already moved away at the baseline, which we confirmed during the follow-up period. We further excluded subjects who reported extreme total energy intake (upper $2.5 \%$ or lower 2.5\%). These exclusions left 18684 eligible men and 20381 women included in this study.

\section{Follow-up and identification of gastric cancer}

Death and change in residence We followed all registered cohort subjects from 1 January 1990 to 31 December 2001. In Japan, all death certificates are submitted to a local government office and forwarded to the PHC in the area of residence. Mortality data are then sent to the Ministry of Health and Welfare and coded for inclusion in the National Vital Statistics. The registration of deaths in Japan is required by the Family Registration Law and is believed to be complete. Therefore, all deaths of cohort subjects were based upon death certificates from each PHC, whenever they stayed in their original area. The changes in residence status were identified annually through the residential registry in each area.

Cancer registry for JPHC study Newly diagnosed cases of cancer were reported by hospitals in and around the study areas when the birth date and residence fulfilled cohort inclusion criteria. Candidate patients were linked by name, address, and date of birth and entered in the cancer registry for the JPHC Study Cohort I. In the Ninohe and Ishikawa PHC areas, a prefecture-wide cancer registry was available (i.e. Iwate and Okinawa Prefecture cancer registry, respectively). Death certificates were used as a supplementary information source for the cancer registry, and 259 cases were first notified by it. As of July 2002, such cases accounted for $7.6 \%$ (DCN (Death Certificate Notification) \%) of all the 3429 entries, which had been diagnosed in 1990-2001. Among them, 39 cases were not confirmed by medical records, and they would account for $1.1 \%$ (DCO (Death Certificate Only) \%) of all the entries.

Identification of gastric cancer Cases of gastric cancer were extracted from the cancer registry for the JPHC Study, based on site (International Classification of Diseases for Oncology (ICD-O) code: $\mathrm{C} 160-169)$ and histological confirmation by biopsy or surgery. A total of 486 cases of gastric cancer, 358 in 18684 men and 128 in 20381 women, were documented as of July 2002 with a histologically proven diagnosis in 1990-2001. This left 10 cases without histological confirmation, which we considered not diagnosed with gastric cancer.

Cardia cancer was defined as a tumour located in the oesophagogastric junction or upper third of the stomach (International Classification of Diseases for Oncology (ICD-O) code C160-161; World Health Organization 1990). A tumour located in the lower side of the stomach was classified as distal gastric cancer (ICD-O code C162-167). Those subsites that could not be classified for its diffuse lesion (ICD-O code C168) or those with no information (ICD-O code C169) were categorised as unclassified. Histological subdivisions were made according to Lauren's (1965) classification.

\section{Statistical analysis}

Person-years at risk were calculated from 1 January 1990 to the date of diagnosed gastric cancer, the date of death, or change in residence or 31 December 2001, whichever came first. A Cox's proportional-hazards model was used to calculate the relative risk (RR) by category of each frequency of dietary intake at baseline after adjusting for covariates using PROC PHREG of the SAS program (SAS Institute, Inc., Cary, NC, USA). Cigarette smoking, fruit and non-green-yellow vegetable intake and age were principally adjusted based on our previous findings (Kobayashi et al, 2002; Sasazuki et al, 2002). Considering the observed colinearity of the intake frequency of three kinds of vegetables (e.g. green, yellow and other vegetables), only nongreen-yellow vegetable intake was included in the multivariate model, because it was more clearly associated with gastric cancer risk (Kobayashi et al, 2002). Although analyses by histological type and by anatomical subsite were conducted, the results are not shown because of unstable findings due to reduced power. 


\section{RESULTS}

Age-adjusted incidence rates per 100000 person-years with number of subjects, person-years of follow-up and number of gastric cancer cases are shown by sex and study area in Table 1, as well as the proportion of each anatomic subsite and histological type. Those rates were three times higher in the men of all three PHC areas except Ishikawa, with similar rates, and differed substantially by $\mathrm{PHC}$ area, especially among men. There were six- and two-fold differences with regard to study area in men and women, respectively. Most tumours $(70 \%$ in men, $75 \%$ in women) were located in the distal portion, and there were no significant differences according to sex or study area. As to histological type, the differentiated type of gastric cancer was predominant in men $(61 \%)$, whereas it was less common in women (37\%). Among men, the proportion of differentiated type was slightly higher in the Saku PHC area and lower in Ishikawa PHC area.

The baseline characteristics according to each quintile category of salt intake are shown in Table 2 . Approximately $60 \%$ of Ishikawa subjects were categorised in the lowest quintile, whereas the majority of Ninohe and Yokote subjects were located in the upper two quintiles. Smokers were more common in upper quintiles of salt intake in men, but they were more common in lower quintiles in women. The intake frequencies of five salted foods were closely correlated with salt intake. The frequencies of fruit and three kinds of vegetable intake were also positively correlated with salt intake, although not remarkable.

The Relative risks and 95\% confidence interval (CI) of each quintile category of salt intakes are shown in Table 3; these were calculated after adjusting for age, cigarette smoking and fruit and vegetable intake. The quintile category of salt intake was dose-

Table I Number of subjects and incident gastric cancer by sex and study area

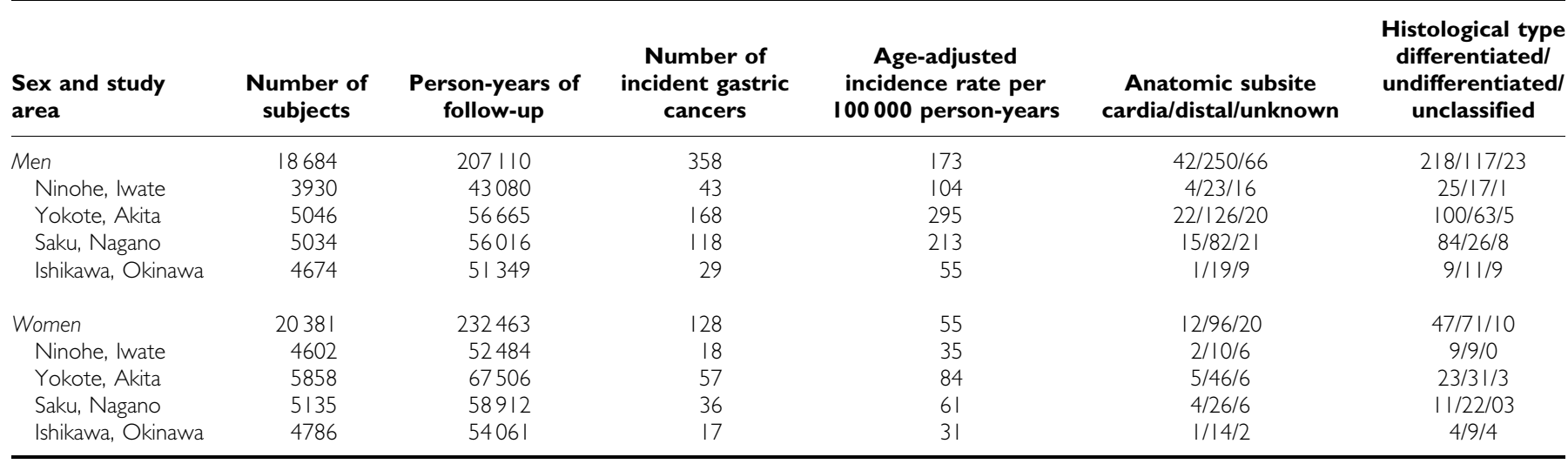

Table 2 Background characteristics of subjects by quintiles of salt intake

\begin{tabular}{|c|c|c|c|c|c|c|c|c|c|c|}
\hline \multirow[b]{2}{*}{ Quintile } & \multicolumn{5}{|c|}{ Men $(n=18684)$} & \multicolumn{5}{|c|}{ Women $(n=2038 \mathrm{I})$} \\
\hline & I (low) & 2 & 3 & 4 & 5 (high) & I (low) & 2 & 3 & 4 & 5 (high) \\
\hline Median $(g / d)^{a}$ & 2.9 & 4.8 & 6.1 & 7.5 & 9.9 & 2.6 & 4.2 & 5.3 & 6.4 & 8.2 \\
\hline \multicolumn{11}{|l|}{ Study area (\%) } \\
\hline Ninohe, Iwate & 8 & 15 & 21 & 27 & 35 & 10 & 16 & 24 & 30 & 33 \\
\hline Yokote, Akita & 13 & 23 & 29 & 33 & 37 & 15 & 24 & 31 & 34 & 39 \\
\hline Saku, Nagano & 19 & 28 & 32 & 32 & 22 & 19 & 30 & 29 & 27 & 21 \\
\hline Age (years) & 48.7 & 49 & 49.4 & 49.9 & 50.1 & 48.9 & 49.3 & 49.7 & 50 & 50 \\
\hline Current smokers (\%) & 49 & 51 & 54 & 56 & 56 & 8.2 & 6.5 & 4.7 & 3.6 & 4.2 \\
\hline Daily alcohol drinkers (\%) & 25 & 34 & 42 & 46 & 47 & 2.1 & 2.7 & 1.8 & 2 & 2.2 \\
\hline \multicolumn{11}{|l|}{ Salted food intake } \\
\hline Dried or salted fish (days week ${ }^{-1}$ ) & 0.9 & 1.5 & 1.9 & 2.3 & 2.9 & 1.1 & 1.8 & 2.1 & 2.4 & 2.9 \\
\hline \multicolumn{11}{|l|}{ Fruit and vegetables } \\
\hline Fruit (days week ${ }^{-1}$ ) & 2.3 & 2.9 & 3.1 & 3.4 & 3.5 & 3.2 & 4 & 4.3 & 4.5 & 4.5 \\
\hline Green vegetables (days week ${ }^{-1}$ ) & 3.2 & 3.4 & 3.6 & 3.6 & 4 & 3.6 & 3.9 & 4 & 4.1 & 4.2 \\
\hline Yellow vegetables (days week ${ }^{-1}$ ) & 2.4 & 2.6 & 2.7 & 2.8 & 2.9 & 3.1 & 3.3 & 3.4 & 3.5 & 3.5 \\
\hline Other vegetables (days week ${ }^{-1}$ ) & 3 & 3.5 & 3.9 & 4.1 & 4.4 & 3.5 & 4.1 & 4.4 & 4.6 & 4.7 \\
\hline
\end{tabular}

${ }^{a}$ Based on food frequency questionnaire. 
Table 3 Multivariate relative risks (RR) of incident gastric cancer by quintiles of salt intake at baseline in men and women: JPHC Study, I990-200 I

\begin{tabular}{|c|c|c|c|c|c|c|}
\hline & \multicolumn{5}{|c|}{ Quintiles of salt intake } & \multirow[b]{2}{*}{$P$ for trend } \\
\hline & I (low) & 2 & 3 & 4 & 5 (high) & \\
\hline \multicolumn{7}{|l|}{ Men } \\
\hline Person-years of follow-up & 40655 & 41445 & 41165 & 41831 & 42016 & \\
\hline No. of incident cancers & 35 & 63 & 73 & 95 & 92 & \\
\hline Age-adjusted incidence rate & 93 & 158 & 179 & 216 & 209 & \\
\hline Multivariate $\mathrm{RR}^{\mathrm{a}}$ & I & 1.74 & 1.96 & 2.3 & 2.23 & \\
\hline $95 \% \mathrm{Cl}$ & Reference & $0.89-2.09$ & $0.88-2.07$ & $1.01-2.34$ & $0.98-2.29$ & 0.08 \\
\hline \multicolumn{7}{|l|}{ Women } \\
\hline Person-years of follow-up & 45828 & 46196 & 46811 & 47097 & 47032 & \\
\hline No. of incident cancers & 23 & 22 & 26 & 19 & 38 & \\
\hline Age-adjusted incidence rate & 52 & 48 & 55 & 39 & 77 & \\
\hline Multivariate $\mathrm{RR}^{\mathrm{a}}$ & I & 0.86 & 0.96 & 0.58 & 1.32 & \\
\hline
\end{tabular}

${ }^{a}$ Adjusted for age in 1990 (40-44, 45-49, 50-54, 55-59 years), cigarette smoking (never, past, current) and fruit and non-green-yellow vegetable intake (almost none, I -2

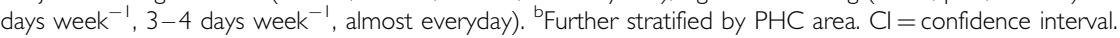

dependently associated with gastric cancer risk in men $(P$ for trend $<0.001)$ and it was attenuated after stratifying by study area $(P$ for trend $=0.08)$. Salt intake was not associated with gastric cancer risk in women.

RR and $95 \%$ CI of intake of five salted food categories are shown in Table 4 for men and Table 5 for women; these were calculated after adjusting for age, cigarette smoking and fruit and vegetable intake. The frequency categories of all listed salted foods were closely associated with the risk in men ( $P$ for trend $<0.05$ ). Although the observed associations were attenuated after further adjusting for salt intake or further stratifying by study area, they were still evident for salted fish roe $(P$ for trend $<0.001$ after adjusting for salt intake and 0.08 after stratifying by study area) and salted fish preserves $(P$ for trend $<0.001$ and 0.03 , respectively). For women, there were no clear associations for intake of miso soup, pickled vegetables and dried or salted fish. However, the associations for salted fish roe and salted fish preserves were clear ( $P$ for trend $=0.003$ for salted fish roe and 0.03 for salted fish preserves) and they were still evident even after further adjusting for salt intake $(P$ for trend $=0.006$ for salted fish roe and 0.06 for salted fish preserves). These associations were still persistent, although not statistically significant, even after stratifying by study area.

\section{DISCUSSION}

In this population-based prospective study of middle-aged Japanese men and women, we observed a dose-dependent increased risks of gastric cancer with the consumption of highly salted foods such as salted fish roe and salted fish preserves. In men, the associations were even clear for estimated total salt and the intake of other salted foods such as miso soup, pickled vegetables and dried or salted fish.

In experimental studies with rats, ingestion of salt is known to cause gastritis, and when coadministered, it enhances the carcinogenic effects of known gastric carcinogens such as $\mathrm{N}$ methyl- $N$-nitro- $N$-nitrosoguanidine (MNNG; Tatematsu et al, 1975; Takahashi and Hasegawa, 1985). Moreover, many epidemiological studies showed that salt or salted food intake increased the risk of gastric cancer (Kono and Hirohata, 1996), including those conducted among Japanese (Tajima and Tominaga, 1985; Hos- hiyama and Sasaba, 1992; Tsugane et al, 1992b). Intragastric highsalt concentration destroys the mucosal barrier, and leads to inflammation and damage such as diffuse erosion and degeneration. The induced proliferous change may enhance the effect of food-derived carcinogens (Takahashi and Hasegawa, 1985). Such mucosal damage also enhances $H$. pylori colonisation in mice (Fox et al, 1999) and possibly in humans (Tsugane et al, 1994), thereby inducing chronic gastritis (Blaser, 1990) and possibly increasing the risk of gastric cancer (International Agency for Research on Cancer, 1994).

The geographic difference observed in this prospective study confirmed our previous findings from an ecological study conducted in the identical study areas in 1989-1990 (Tsugane et al, 1991). The male age-standardised mortality rate (world population) per 100000 population for $1985-1987$ were 29.7 in Ninohe, 49.4 in Yokote, 42.8 in Saku and 17.2 in Ishikawa, respectively. In that study, average amount of salt excretion in 24-h urine $\left(\mathrm{g} \mathrm{day}^{-1}\right)$ from randomly selected men aged $40-49$ years was closely associated $\left(r^{2}=0.995\right)$ with the gastric cancer mortality in these areas: 9.9 in Ninohe, 13.4 in Yokote, 11.9 in Saku and 8.0 in Ishikawa. However, this strong correlation was largely attenuated $\left(r^{2}=0.265\right)$ with average amounts of dietary salt intake $\left(\mathrm{g} \mathrm{day}^{-1}\right)$ based on 3-day dietary record and calculated from the Standard Tables of Food Composition (Science and Technology Agency, 1982): 15.5 in Ninohe, 15.0 in Yokote, 13.2 in Saku and 12.0 in Ishikawa. The average amount of estimated salt intake $\left(\mathrm{g} \mathrm{day}^{-1}\right)$ in this study with limited food items was based on the standard composition table (Science and Technology Agency, 1982): 7.7 in Ninohe, 7.3 in Yokote, 6.6 in Saku and 4.5 in Ishikawa. Therefore, the estimated salt intake was relatively overestimated in Ninohe and may have underestimated the true association between salt intake and gastric cancer incidence, especially in women. The precise estimation of salt intake may be implausible especially in different study areas, where the content of salt may be differed in each area even for the same food item. Although multiple 24-h urine collections may be an ideal method to estimate habitual salt intake (Liu et al, 1979), they are not feasible for a large-scale prospective study.

The observed weak link between salt intake and gastric cancer in women may also be due to relatively low validity of estimated salt intake (Tsubono et al, 2003). The Spearman rank correlation with 28 -day dietary record was 0.54 in women, however, that with 2 -day 
Table 4 Relative risks (RR) of incident gastric cancer by category of salted food intake at baseline in men: JPHC Study, 1990-200।

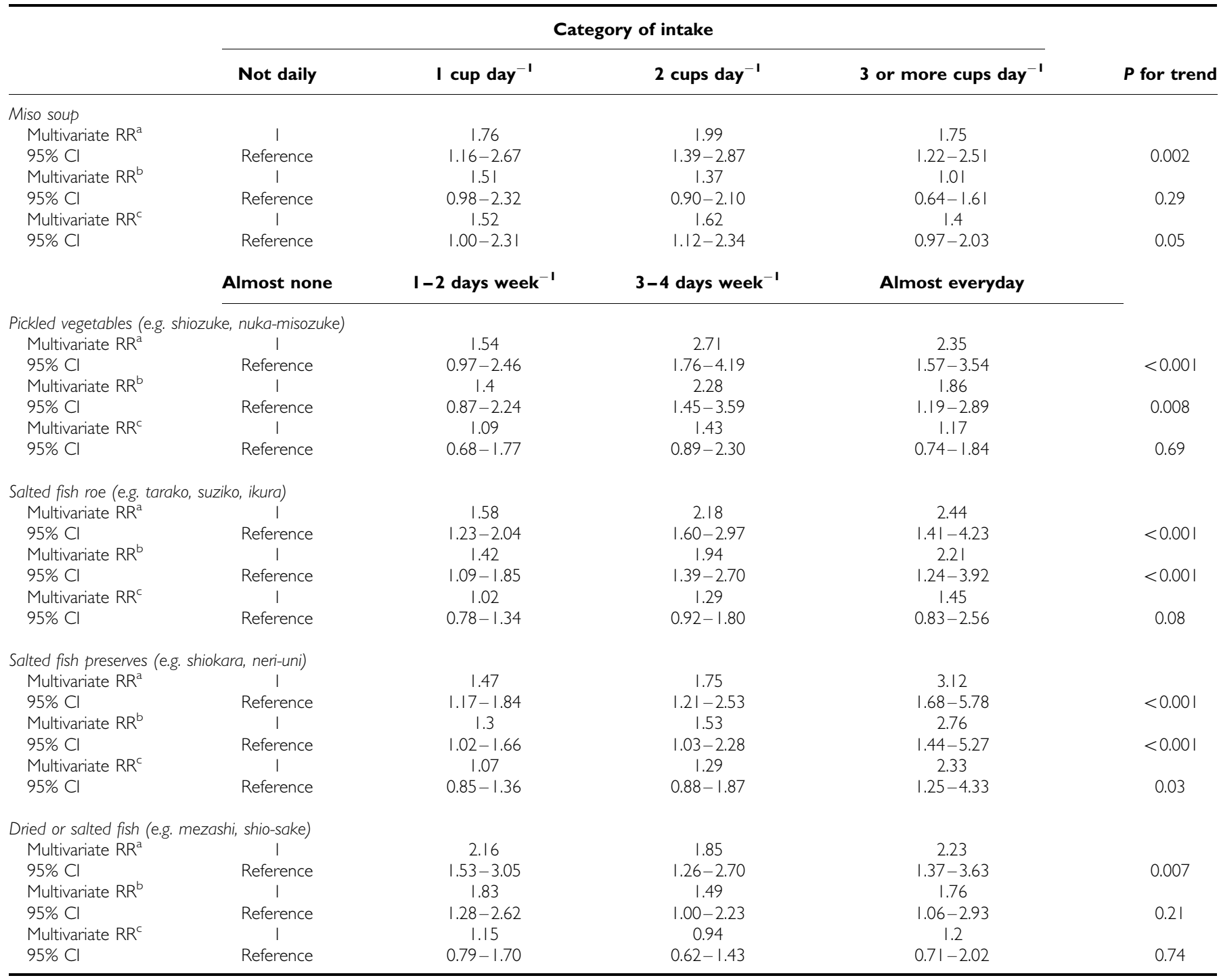

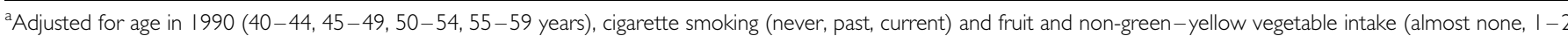

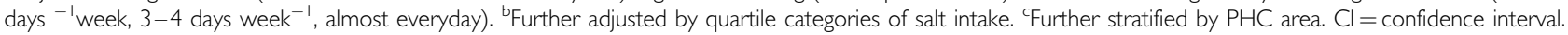

urinary excretion was only 0.12 . The corresponding values in men were 0.49 and 0.38 , respectively.

The associations with salt and salted food intake were always attenuated after stratifying by study area, where both salt/salted food intake and gastric cancer incidence varied significantly and were well correlated at the population level. Owing to the difficulties in estimating the intake of salt per se, its validity is expected to be lower within each study area with limited variation when compared with that in four areas combined. Therefore, stratification by study area may have underestimated the true association. Nonetheless, the associations were still clear with salt intake in men.

Among the salted foods, consumption of highly salted food such as salted fish roe and salted fish preserves were strongly associated with an increased risk of gastric cancer, even when adjusted for salt intake and stratified by study area both in men and women. The salt content of these salted foods is more than $5 \%$; that of other salted foods such as miso soup, pickled vegetables and dried fish varied widely, but it is less than $5 \%$ in most foods. The observed findings might imply that either highly salted fish such as salted roe or salted preserves itself increased the risk of gastric cancer or that its intake was merely a good marker for a preference for salted foods or salt intake in general. Since 'almost daily' consumers of salted fish roe $(2.2 \%)$ or salted fish preserves $(0.9 \%)$ were limited, the intake frequency of these foods served to effectively categorise the subjects according to their preference for salted food intake. However, the intake frequencies of miso soup $(77 \%)$ or pickled vegetables $(49 \%)$ were relatively common, and their salt contents are different in each serving and each individual. Salt content of miso collected from 39 regions in 20 prefectures across Japan showed a wide variation, from 9.1 to $18.2 \%$ on the regional average (Watanabe et al, 1982) and those in miso soup preparation ( $18 \mathrm{~g}$ miso $200 \mathrm{~g}^{-1}$ soup in general recipe) varied individually. Salt contents of pickled vegetables also varied from less than $1 \%$ to over $10 \%$. Even miso soup or pickled vegetables may have increased the risk of gastric cancer among subjects who generally consumed these types of foods with relatively higher salt content. An alternative explanation for the strong associations between highly salted foods and gastric cancer may be due to chemical caricinogens, which can be formed by reacting nitrate or nitrite in the process of preservation and of digestion in the stomach. 
Table 5 Relative risks (RR) of incident gastric cancer by category of salted food intake at baseline in women: JPHC Study, 1990-200।

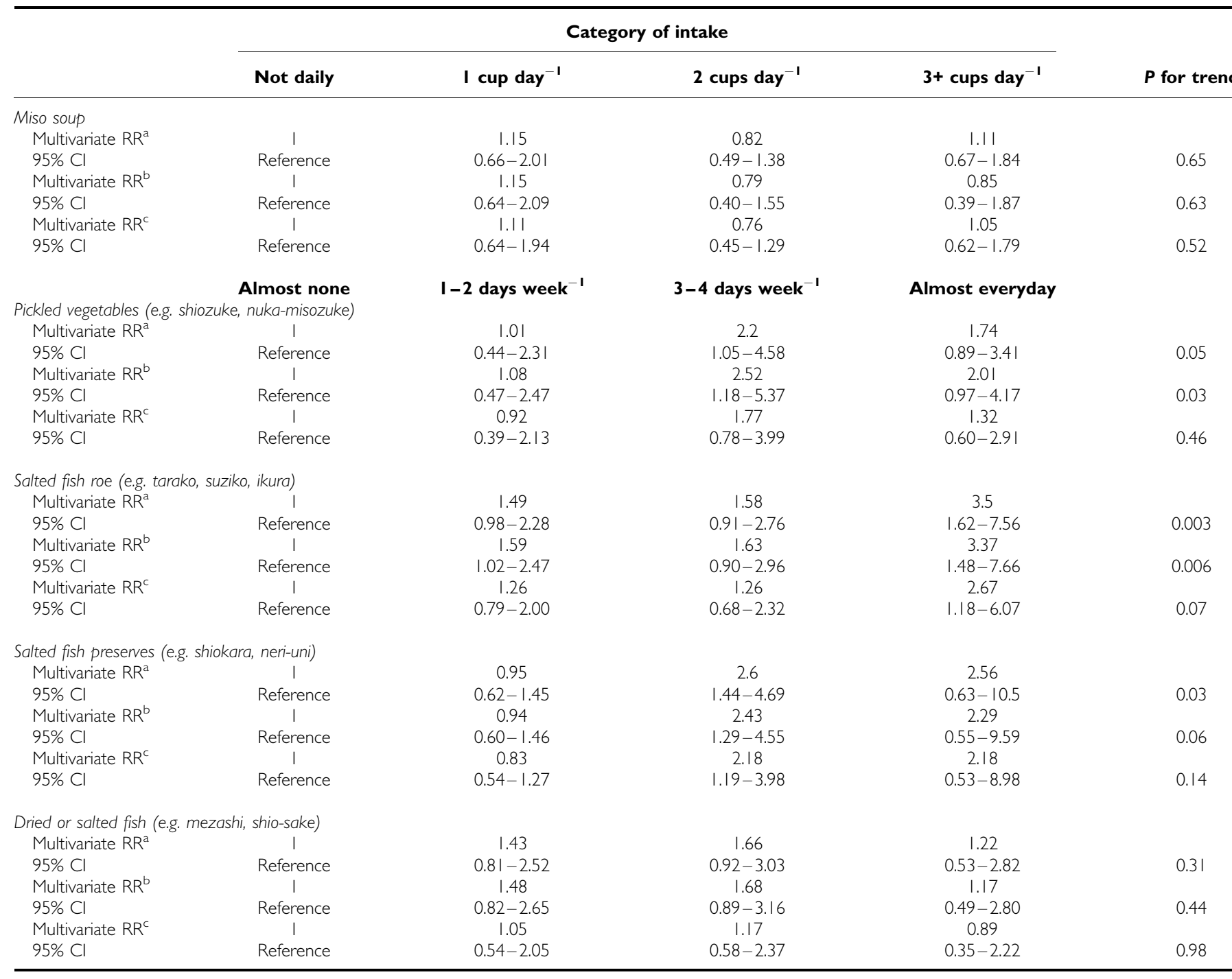

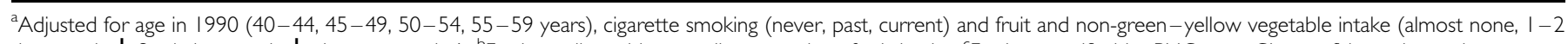
days week ${ }^{-1}, 3-4$ days week $^{-1}$, almost everyday). ${ }^{b}$ Further adjusted by quartile categories of salt intake. ${ }^{c}$ Further stratified by $\mathrm{PHC}$ area. $\mathrm{Cl}=$ confidence interval.

Helicobacter pylori infection is closely associated with the risk of gastric cancer (Huang et al, 1998; Helicobacter and Cancer Collaborative Group 2001). The prevalence of $H$. pylori IgG antibody among randomly selected men aged $40-49$ years was $76 \%$ in Ninohe, $86 \%$ in Yokote, $72 \%$ in Saku and $63 \%$ in Ishikawa PHC areas in our previous ecological study in 1989-1990 (Tsugane et al, 1994), which nearly paralleled age-adjusted incidence rates of gastric cancer in each area (Table 1). However, consumption of salted fish roe and salted fish preserves in these male cohort subjects was also closely associated with gastric cancer incidence at the population level: 5.8 and 4.3 days month $^{-1}$ in Ninohe, 7.9 and 4.9 days month ${ }^{-1}$ in Yokote, 4.3 and 3.8 days month ${ }^{-1}$ in Saku and 0.9 and 1.0 days month ${ }^{-1}$ in Ishikawa (Tsugane et al, 2001). Moreover, a cross-sectional analysis in our ecological study showed that the intake of both pickled vegetable and miso soup was associated with $H$. pylori infection (Tsugane et al, 1994), although salted fish roe and salted fish preserves were not assessed in that study. An infection with H. pylori, however, is itself unlikely to increase the intake of salted foods. Therefore, even if $H$. pylori infection causes gastric cancer, restriction of salt and salted food intake can at least reduce its risk. It should be acknowledged that the worldwide decrease in the age-adjusted incidence of gastric cancer was obviously not due to the intentional eradication of
$H$. pylori infection, but can be related to a reduction in salted food intake by use of refrigerators (World Cancer Research Fund in association with American Institute for Cancer Research, 1997). However, it is possible that improvement of sanitary condition and familial crowding may have been associated with unintentional falls in the prevalence of $H$. pylori infection and consequently gastric cancer incidence in recent birth cohorts especially in developed countries (Taylor and Blaser, 1991; International Agency for Research on Cancer, 1994).

To summarise, the intake of salt and salted food, especially highly salted food, was closely associated with the risk of gastric cancer. Considering that a substantial number of Japanese consume such foods daily and the relatively high risk involved, the restriction of these foods may be a practical way to prevent gastric cancer.

\section{ACKNOWLEDGEMENTS}

This study was supported by Grants-in-aid for cancer research and for the second term comprehensive 10-year strategy for cancer control from the Ministry of Health, Labor and Welfare of Japan. We thank all staff members in each study area and in the central office for their painstaking efforts to conduct the baseline survey and follow-up. 


\section{REFERENCES}

Blaser MJ (1990) Helicobacter pylori and the pathogenesis of gastroduodenal inflammation. I Infect Dis 161: 626-633

Fox JG, Dangler CA, Taylor NS, King A, Koh TJ, Wang TC (1999) High-salt diet induces gastric epithelial hyperplasia and parietal cell loss, and enhances Helicobacter pylori colonization in C57BL/6 mice. Cancer Res 59: $4823-4828$

Helicobacter and Cancer Collaborative Group (2001) Gastric cancer and Helicobacter pylori: a combined analysis of 12 case-control studies nested within prospective cohorts. Gut 49: 347-353

Hoshiyama Y, Sasaba T (1992) A case-control study of stomach cancer and its relation to diet, cigarettes, and alcohol consumption in Saitama Prefecture, Japan. Cancer Causes Control 3: 441-448

Huang JQ, Sridhar S, Chen Y, Hunt RH (1998) Meta-analysis of the relationship between Helicobacter pylori seropositivity and gastric cancer. Gastroenterology 114: 1169-1179

International Agency for Research on Cancer (1994) Schistosomes, Liver Flukes, and Helicobacter pylori, IARC Monographs on the Evaluation of Carcinogenic Risks to Humans, Vol. 61, Lyon: International Agency for Research on Cancer

Joossens IV, Hill MJ, Elliott P, Stamler R, Lesaffre E, Dyer A, Nichols R, Kesteloot H (1996) Dietary salt, nitrate and stomach cancer mortality in 24 countries. European Cancer Prevention (ECP) and the INTERSALT Cooperative Research Group. Int J Epidemiol 25: 494- 504

Kato I, Tominaga S, Matsumoto K (1992) A prospective study of stomach cancer among a rural Japanese population: a 6-year survey. Jpn J Cancer Res 83: $568-575$

Kneller RW, McLaughlin JK, Bjelke E, Schuman LM, Blot WJ, Wacholder S, Gridley G, CoChien HT, Fraumeni Jr JF (1991) A cohort study of stomach cancer in a high-risk American population. Cancer 68: 672-678

Kobayashi M, Tsubono Y, Sasazuki S, Sasaki S, Tsugane S, for JPHC Study Group (2002) Vegetables, fruit, and risk of gastric cancer in Japan: a 10year follow-up of the JPHC Study Cohort I. Int J Cancer 102: 39-44

Kono S, Hirohata T (1996) Nutrition and stomach cancer. Cancer Causes Control 7: $41-55$

Lauren P (1965) The two histological main types of gastric carcinoma: diffuse and so-called intestinal-type carcinoma. APMIS 64: $31-49$

Liu K, Cooper R, McKeever J, McKeever P, Byington R, Soltero I, Stamler R, Gosch F, Stevens E, Stamler J (1979) Assessment of the association between habitual salt intake and high blood pressure: methodological problems. Am J Epidemiol 110: 219-226

Nomura A, Grove JS, Stemmermann GN, Severson RK (1990) A prospective study of stomach cancer and its relation to diet, cigarettes, and alcohol consumption. Cancer Res 50: 627-631

Research Group for Population-based Cancer Registration in Japan (2002) Cancer incidence and incidence rates in Japan in 1997: estimates based on data from nine population-based cancer registries. Jpn J Clin Oncol 32: $318-322$

Sasazuki S, Sasaki S, Tsugane S, for the JPHC Study Group (2002) Cigarette smoking, alcohol consumption, and subsequent gastric cancer risk by subsite and histologic type. Int J Cancer 101: 560-566

Science and Technology Agency (1982) Standard Tables of Food Composition in Japan, 4th rev. edn., Tokyo: Printing Bureau, Ministry of Finance

Tajima K, Tominaga S (1985) Dietary habits and gastro-intestinal cancers: a comparative case-control study of stomach and large intestinal cancers in Nagoya, Japan. Jpn J Cancer Res 76: 705-716

\section{Appendix}

The investigators and participating institutions in the JPHC Study Cohort I Group, a part of JPHC Study Group (Principal investigator: S Tsugane), were as follows: S Tsugane, S Sasaki, Epidemiology and Biostatistics Division, National Cancer Center Research Institute East, Kashiwa; J Ogata, S Baba, National Center for Circulatory Diseases, Suita; K Miyakawa, F Saito, A Koizumi, Iwate Prefectural Ninohe Public Health Center, Ninohe; Y Miyajima, N Suzuki, S Nagasawa; Akita Prefectural Yokote Public Health Center, Yokote; H Sanada, Y Hatayama, F Kobayashi, H Uchino, Y Shirai, T Kondo, Nagano Prefectural Saku Public Health Center, Saku; Y Kishimoto, E Takara, M Kinjo,
Takahashi M, Hasegawa R (1985) Enhancing effects of dietary salt on both initiation and promotion stages of rat gastric carcinogenesis. Princess Takamatsu Symp 16: 169-182

Tatematsu M, Takahashi M, Fukushima S, Hananouchi M, Shirai T (1975) Effects in rats of salt on experimental gastric cancers induced by $\mathrm{N}$ methyl- $N$-nitro- $N$-nitrosoguanidine or 4-nitroquinoline-1-oxide. J Natl Cancer Inst 55: $101-106$

Taylor DN, Blaser MJ (1991) The epidemiology of Helicobacter pylori infection. Epidemiol Rev 13: 42 - 59

Tsubono Y, Kobayashi M, Sasaki S, Tsugane S (2003) Validity and reproducibility of a self-administered food frequency questionnaire used in the baseline survey of the JPHC Study Cohort I. J Epidemiol 13: S125-S133

Tsubono Y, Kobayashi M, Tsugane S (1997) Food consumption and gastric cancer mortality in five regions of Japan. Nutr Cancer 27: 60-64

Tsugane S, Akabane M, Inami T, Matsushima S, Ishibashi T, Ichinowatari Y, Miyajima Y, Watanabe S (1991) Urinary salt excretion and stomach cancer mortality among four Japanese populations. Cancer Causes Control 2: $165-168$

Tsugane S, Fahey MT, Sasaki S, Baba S (1999) Alcohol consumption and allcause and cancer mortality among middle-aged Japanese men: seven-year follow-up of the JPHC study Cohort I. Am J Epidemiol 150: $1201-1207$

Tsugane S, Gey F, Ichinowatari Y, Miyajima Y, Ishibashi T, Matsushima S, Hirota Y, Ianami T, Yamaguchi M, Karita K, Kabuto M, Takashima Y, Todorik H, Tsuda M, Akabane M, Furaichi Y, Hamada G, Watanabe S (1992a) Cross-sectional epidemiologic study for assessing cancer risks at the population level. I. Study design and participation rate. J Epidemiol 2: $75-81$

Tsugane S, Sasaki S, Kobayashi M, Tsubono Y, Akabane M (2003) Validity and reproducibility of the self-administered food frequency questionnaire in the JPHC Study Cohort I: study design, conduct and participant profiles. J Epidemiol 13: S2-S12

Tsugane S, Sasaki S, Kobayashi M, Tsubono Y, Sobue T (2001) Dietary habits among JPHC Study participants at baseline survey. J Epidemiol 11: $\mathrm{S} 30-\mathrm{S} 43$

Tsugane S, Sobue T (2001) Baseline survey of JPHC Study - design and participation rate. J Epidemiol 11: S24-S29

Tsugane S, Tei Y, Takahashi T, Watanabe S, Sugano K (1994) Salty food intake and risk of Helicobacter pylori infection. Jpn J Cancer Res 85: 474 478

Tsugane S, Tsuda M, Gey F, Watanabe S (1992b) Cross-sectional study with multiple measurements of biological markers for assessing stomach cancer risks at the population level. Environ Health Perspect 98: 207-210

Watanabe T, Miyasaka M, Koizumi A, Ikeda M (1982) Regional difference in salt content in home-made and store-bought preparations of miso paste. Tohoku J Exp Med 137: 305-313

World Cancer Research Fund in association with American Institute for Cancer Research (1997) Food, Nutrition and the Prevention of Cancer: A Global Perspective. Washington, DC: American Institute for Cancer Research

World Health Organization (1990) International Classification of Diseases for Oncology, 2nd edn., Geneva: World Health Organization

World Health Organization (1997) The World Health Report 1997. Geneva: World Health Organization

World Health Organization (2003) Diet, Nutrition and the Prevention of Chronic Diseases, WHO Technical Report Series 916, Geneva: World Health Organization

T Fukuyama, Okinawa Prefectural Ishikawa Public Health Center, Ishikawa; S Matsushima, S Natsukawa, Saku General Hospital, Usuda; S Watanabe, M Akabane, Tokyo University of Agriculture, Tokyo; M Konishi, Ehime University, Matsuyama; $S$ Tominaga, Aichi Cancer Center Research Institute, Nagoya; M Iida, S Sato, Center for Adult Diseases, Osaka; the late M Yamaguchi, Y Matsumura, National Institute of Health and Nutrition, Tokyo; Y Tsubono, Tohoku University, Sendai; H Iso, Tsukuba University, Tsukuba; H Sugimura, Hamamatsu University, Hamamatsu and M Kabuto, National Institute for Environmental Studies. 